\title{
The Primitivity and Primitive Exponents of a Class of Nonnegative Matrix Pairs
}

\author{
Meijin Luo \\ School of Mathematics and Statistics, Hechi University Yizhou, Guangxi 546300, P.R. China; \\ meijin322@163.com
}

Keywords: Upper bound; Nonnegative; Matrix pairs; Two-Colored digraph; Exponent.

Abstract. It is a brand-new research in combinatorial matrix theory to extend the exponent of traditional single nonnegative primitive matrix to the exponent of nonnegative primitive matrix pairs. With the knowledge of graph theory, the problem of primitive exponent of nonnegative matrix pairs can be transformed into the associated directed digraph of nonnegative matrix pairs, that is two-colored digraphs. A class of two-colored digraphs whose uncolored digraph has $4 n+2$ vertices and consists of one $(4 n+1)$-cycle and one $n$-cycle is considered. The primitive conditions, the upper bound, the lower bound, and the characterizations of extremal two-colored digraphs are given. The results provide a basis for the study of the exponent of nonnegative primitive matrix pairs and the exponent of nonnegative primitive matrix tuples in the general case.

\section{Introduction}

Let $D$ be a digraph. A walk in $D$ of length $l$ is a sequence $v_{1}, v_{2}, \cdots, v_{l+1}$ of vertices such that there is an arc in $D$ from $v_{i}$ to $v_{i+1}$ for $i=1,2, \cdots, l$. The walk is a path if the vertices $v_{1}, v_{2}, \cdots, v_{l+1}$ are distinct[1]. A two-colored digraph is a digraph whose arcs are colored red and blue. We allow loops and both a red arc and blue arc from $i$ to $j$ for all pairs $(i, j)$ of vertices. $D$ is strongly connected provided for each pair $(i, j)$ of vertices there is a walk in $D$ from $i$ to $j$. Given a walk $\omega$ in $D, r(\omega)$ (respectively, $b(\omega)$ ) is the number of red arcs (respectively, blue arcs) of $\omega$, and the composition of $\omega$ is the vector $(r(\omega), b(\omega))$ or $(r(\omega), b(\omega))^{T}$.

A two-colored directed digraph $D$ is primitive if and only if there exist nonnegative integers $h$ and $k$ with $h+k>0$ such that for each pair $(i, j)$ of vertices there is a $(h, k)$-walk in $D$ from $i$ to $j$. A $(h, k)$-walk from $i$ to $j$ consisting of $h$ red arcs and $k$ blue arcs. The exponent of the primitive two-colored digraph $D$, denoted $\exp (D)$, is defined to be the smallest value of $h+k$ over all such $h$ and $k$.

Let $C=\left\{\gamma_{1}, \gamma_{2}, \cdots, \gamma_{l}\right\}$ be the set of cycles of $D$. Set $M$ to be the $2 \times l$ matrix whose $i$ th column is the composition of $\gamma_{i}$. We call $M$ the cycle matrix of $D$. The content of $M$, denoted content $(M)$, is defined to be 0 if the rank of $M$ is less than 2 and the greatest common divisor of all $2 \times 2$ minors of $M$, otherwise.

Lemma 1.1 Let $D$ be a two-colored digraph having at least one red arc and one blue arc. Then $D$ is primitive if and only if $D$ is strongly connected and content $(M)=1[2]$.

In fact, there is a one-to-one relationship between nonnegative matrix pairs and two-colored digraphs.By studying the exponent of associated directed digraph of nonnegative matrix pairs, that is two-colored directed digraphs, the problem of nonnegative matrix, the upper bound and the lower bound of primitive exponent, the characterizations of extremeal digraphs etc can be solved.Some results have already been obtained [4-10].

In this paper, for $n \geq 2$, we consider the two-colored digraph $D$ in Figure. 1 with $4 n+2$ vertices which has at least one red and one blue arc. 


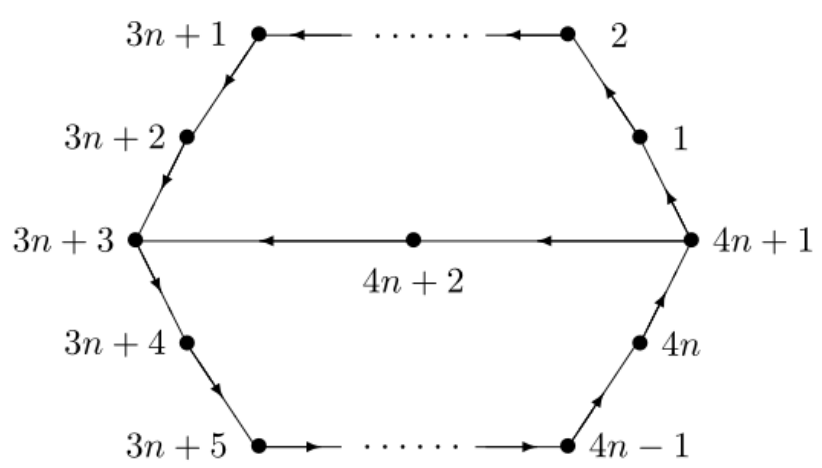

Figure 1. Uncolored digraph of $D$

Clearly, $D$ has only two cycles that one is an $(4 n+1)$-cycle and the other one is an $n$-cycle. Without loss of generality, we can assume that the cycle matrix of $D$ is

$$
M=\left[\begin{array}{cc}
x & y \\
4 n+1-x & n-y
\end{array}\right]
$$

for some nonnegative integers $x$ and $y$ with $\frac{4 n+1}{2} \leq x \leq 4 n+1$.

\section{The Primitivity}

Theorem 2.1 Let $D$ be primitive. Then if and only if $x=4 n-3, y=n-1$.

Proof From $(1), \operatorname{det}(M)=n x-(4 n+1) y$. By Lemma 1.1, $D$ is primitive if and only if content $(M)$ $=1$, that is, $\operatorname{det}(M)= \pm 1$. So $n x-(4 n+1) y= \pm 1$. Because $\frac{4 n+1}{2} \leq x \leq 4 n+1$, we have $x=4 n-3, y=n-1$, then the theorem follows.

By Theorem 2.1, we obtain the $(4 n+1)$-cycle of $D$ contains four blue arcs, and the $n$-cycle of $D$ contains exactly one blue arc, the cycle matrix of $D$ is

And the inverse matrix of $M$ is

$$
M=\left[\begin{array}{cc}
4 n-3 & n-1 \\
4 & 1
\end{array}\right]
$$

$$
M^{-1}=\left[\begin{array}{cc}
1 & -(n-1) \\
-4 & 4 n-3
\end{array}\right]
$$

\section{The Bounds on the Exponents}

Theorem 3.1 Let $D$ be primitive. Then $8 n^{2}+9 n+1 \leq \exp (D) \leq 32 n^{2}-20 n-3$.

Proof We first prove $\exp (D) \leq 32 n^{2}-20 n-3$.

We only prove that between each pair $(i, j)$ of vertices of $D$ there is an $\left(36 n^{2}-52 n+21,32 n-\right.$ 24) -walk. For any pair $(i, j)$ vertices of $D$, let $P_{i j}$ be the shortest path in $D$ from $i$ to $j$, and denote $r\left(P_{i j}\right)=r$ and $b\left(P_{i j}\right)=b$. We see that

$$
\left[\begin{array}{l}
r \\
b
\end{array}\right]+(4 n-3-r+(n-1) b)\left[\begin{array}{c}
4 n-3 \\
4
\end{array}\right]+(16 n-12+4 r-(4 n-3) b)\left[\begin{array}{c}
n-1 \\
1
\end{array}\right]=\left[\begin{array}{c}
36 n^{2}-52 n+21 \\
32 n-24
\end{array}\right] \text {. }
$$

Noting that $0 \leq r \leq 4 n-3,0 \leq b \leq 5$, it is easy to see that $4 n-3-r+(n-1) b \geq 0$ and $16 n-12+$ $4 r-(4 n-3) b \geq 0$. If $r=4 n-3$, then $b \geq 0$ and if $b=4$, then $r \geq 0$. If $b=5$, then the walk that starts at vertex $i$, follows $P_{i j}$ to vertex $j$, goes one time around the $(4 n+1)$-cycle at least. This gives

$$
\exp (D) \leq 36 n^{2}-52 n+21+32 n-24=32 n^{2}-20 n-3 .
$$

We next prove $\exp (D) \geq 8 n^{2}+9 n+1$.

Suppose that $(h, k)$ is a pair of nonnegative integers such that for all pairs $(i, j)$ of vertices there is 
an $(h, k)$-walk from $i$ to $j$. By considering $i=j=4 n+1$, we see that there exist nonnegative integers $u$ and $v$ with

$$
\left[\begin{array}{l}
h \\
k
\end{array}\right] M=\left[\begin{array}{l}
u \\
v
\end{array}\right] .
$$

From Figure. 1 and the cycle matrix of $D$, we can see there is at least an $n+1$ red path on the $(4 n+1)$-cycle. Taking $i$ and $j$ to be the initial vertex and the terminal vertex of the $n+1$ red path on the $(4 n+1)$-cycle, and this path has composition $(n+1,0)$.Hence

$$
M z=\left[\begin{array}{c}
h-(n+1) \\
k
\end{array}\right]
$$

has a nonnegative integer solution. Necessarily,

$$
z=M^{-1}\left[\begin{array}{c}
h-(n+1) \\
k
\end{array}\right]=\left[\begin{array}{l}
u \\
v
\end{array}\right]-M^{-1}\left[\begin{array}{c}
n+1 \\
0
\end{array}\right]=\left[\begin{array}{l}
u \\
v
\end{array}\right]-\left[\begin{array}{c}
n+1 \\
-4 n-4
\end{array}\right] \geq 0
$$

So $u \geq n+1$. Next take $i$ and $j$ to be the terminal vertex and the initial vertex of the $n+1$ red path on the $(4 n+1)$-cycle, and this path has composition $(3 n-4,4)$.Hence

$$
M z=\left[\begin{array}{c}
h-(3 n-4) \\
k-4
\end{array}\right]
$$

has a nonnegative integer solution. Necessarily,

$$
z=M^{-1}\left[\begin{array}{c}
h-(3 n-4) \\
k-4
\end{array}\right]=\left[\begin{array}{l}
u \\
v
\end{array}\right]-M^{-1}\left[\begin{array}{c}
3 n-4 \\
4
\end{array}\right]=\left[\begin{array}{l}
u \\
v
\end{array}\right]-\left[\begin{array}{c}
-n \\
4 n+4
\end{array}\right] \geq 0 .
$$

So $v \geq 4 n+4$.Thus

$$
h+k=\left[\begin{array}{ll}
1 & 1
\end{array}\right] M\left[\begin{array}{l}
u \\
v
\end{array}\right] \geq\left[\begin{array}{ll}
4 n+1 & n
\end{array}\right]\left[\begin{array}{c}
n+1 \\
4 n+4
\end{array}\right]=8 n^{2}+9 n+1 .
$$

Thus the theorem follows.

\section{The Extremeal Two-Colored Digraphs}

Lemma 4.1 Let $D$ be primitive. If there is exactly four red paths $\omega_{1}, \omega_{2}, \omega_{3}$, and $\omega_{4}$ of length $n-1$, $n-1, n-2$ and $n+1$ on the $(4 n+1)$-cycle, that is, there is the longest red path of length $n+1$ on the $(4 n+1)$-cycle, then $\exp (D)=8 n^{2}+9 n+1$.

Proof Form Theorem 3.1, we only proof $\exp (D) \leq 8 n^{2}+9 n+1$.

For any pair $(i, j)$ of vertices of $D$, let $P_{i j}$ be the shortest path in $D$ from $i$ to $j$, and denote $r\left(P_{i j}\right)=r$ and $b\left(P_{i j}\right)=b$. We see that

$$
\left[\begin{array}{l}
r \\
b
\end{array}\right]+(n+1-r+(n-1) b)\left[\begin{array}{c}
4 n-3 \\
4
\end{array}\right]+(4 n+4+4 r-(4 n-3) b)\left[\begin{array}{c}
n-1 \\
1
\end{array}\right]=\left[\begin{array}{c}
8 n^{2}+n-7 \\
8 n+8
\end{array}\right]
$$

We consider the following six cases:

Case $1: b=0$.

It is clear that $0 \leq r \leq n+1$. Hence $n+1-r \geq 0$ and $4 n+4+4 r>0$. By (2), the walk that starts at vertex $i$, follows $P_{i j}$ to vertex $j$, goes $n+1-r$ times around the $(4 n+1)$-cycle, and $4 n+4+4 r$ times around the $n$-cycle is an $\left(8 n^{2}+n-7,8 n+8\right)$-walk from $i$ to $j$.

Case $2: b=1$.

It is clear that $0 \leq r \leq 2 n$. Hence $2 n-r \geq 0$ and $4 r+7>0$.By (2), the walk that starts at vertex $i$, follows $P_{i j}$ to vertex $j$, goes $2 n-r$ times around the $(4 n+1)$-cycle, and $4 r+7$ times around the $n$ -cycle is an $\left(8 n^{2}+n-7,8 n+8\right)$-walk from $i$ to $j$.

Case $3: b=2$.

It is clear that $n-2 \leq r \leq 3 n-1$. Hence $3 n-1-r \geq 0$ and $-4 n+4 r+10>0$. By (2), the walk that 
starts at vertex $i$, follows $P_{i j}$ to vertex $j$, goes $3 n-1-r$ times around the $(4 n+1)$-cycle, and

$-4 n+4 r+10$ times around the $n$-cycle is an $\left(8 n^{2}+n-7,8 n+8\right)$-walk from $i$ to $j$.

Case $4: b=3$.

It is clear that $2 n-3 \leq r \leq 4 n-3$. Hence $4 n-2-r>0$ and $-8 n+4 r+13>0$. By (2), the walk that starts at vertex $i$, follows $P_{i j}$ to vertex $j$, goes $4 n-2-r$ times around the $(4 n+1)$-cycle, and $-8 n+4 r+13$ times around the $n$-cycle is an $\left(8 n^{2}+n-7,8 n+8\right)$-walk from $i$ to $j$.

Case $5: b=4$.

It is clear that $3 n-4 \leq r \leq 4 n-3$. Hence $5 n-3-r>0$ and $-12 n+4 s+16 \geq 0$. By (2), the walk that starts at vertex $i$, follows $P_{i j}$ to vertex $j$, goes $5 n-3-r$ times around the $(4 n+1)$-cycle, and $-12 n+4 r+16$ times around the $n$-cycle is an $\left(8 n^{2}+n-7,8 n+8\right)$-walk from $i$ to $j$.

Case 6: $b=5$.

It is clear that $4 n-3 \leq r \leq 5 n-2$. Hence $6 n-4-r>0$ and $-16 n+4 r+19>0$.By (2), the walk that starts at vertex $i$, follows $P_{i j}$ to vertex $j$, goes $6 n-4-r$ times around the $(4 n+1)$-cycle, and $-16 n+4 r+19$ times around the $n$-cycle is an $\left(8 n^{2}+n-7,8 n+8\right)$-walk from $i$ to $j$.

Then $\exp (D) \leq 8 n^{2}+n-7+8 n+8=8 n^{2}+9 n+1$. The lemma follows.

As the proof of Theorem 4.1, we have the following Lemma.

Lemma 4.2 Let $D$ be primitive.If and only if there is exactly one red path $\omega$ of length $4 n-3$ on the $(4 n+1)$-cycle, then $\exp (D)=32 n^{2}-20 n-3$.

\section{Acknowledgements}

This research was supported by Project of Guangxi Colleges and Universities (NO.YB2014335) and Scientific and Technologial Innovation Programs of Higher Education Institutions in Shanxi (NO.20151113).

\section{References}

[1] R.A. Brualdi, H.J. Ryser, Combinatorial Matrix Theory, Encyclopedia of Mathematics and its Applications, vol. 39, Cambridge University Press, Cambridge, 1991.

[2] B.L. Shader, S. Suwilo, Exponents of nonnegative matrix pairs, Linear Algebra Appl, vol. 363(2003), pp. 275-293.

[3] A. Berman, R. Plemmons, Nonnegative Matrices in the Mathematical Science, Classics in Applied Mathematics. Vol. 9, SIAM, Philadelphia, PA, 1994.

[4] Y.B. Gao, Y.L. Shao, Exponents of two-colored digraphs with two cycles, Linear Algebra Appl, 407(2005), pp. 263-270.

[5] Y.L. Shao, Y.B. Gao, L. Sun, Exponents of a class of two-colored digraphs, Linear and Multilinear Algebra,53:3 (2005), pp. 175-188.

[6] M.J. Luo. The primitive sets of a class of two-colored digraphs with one common vertex, Journal of Jinan University of China: Natural Sciences, 34:5 (2013), pp. 483-488. (In Chinese)

[7] M.J. Luo. The primitive sets of a class of two-colored digraphs, Mathematics in Practice and Theory, 42:24 (2012), pp. 253-258. (In Chinese)

[8] Q. Yan, Y.L. Shao, exponents of a class of three-colored digraphs, Journal of North University of China(Natural Science), 30:6 (2009), pp. 512-517.(In Chinese)

[9] M.J. Luo, Y.B. Gao, Primitive exponents of a class of three-colored digraphs with three cycles, Journal of Shandong University (Natural Science), 43:1 (2008), pp. 65-72.(In Chinese)

[10]H.L. Zhou, Q.Y. Song, exponents of a class of three-colored digraphs, Journal of Jilin Normal University(Natural Science), 5:2 (2011), pp. 82-89.(In Chinese) 